\title{
Molecular Characterization and Expression of PFT, an FHB Resistance Gene at the Fhb1 QTL in Wheat
}

\author{
Yi He, Xu Zhang, Yu Zhang, Dawood Ahmad, Lei Wu, Peng Jiang, and Hongxiang Ma†
}

\begin{abstract}
All authors: Institute of Food Crops, Jiangsu Academy of Agricultural Sciences/Jiangsu Collaborative Innovation Center for Modern Crop Production, Nanjing, China; and fourth author: Institute of Biotechnology and Genetic Engineering, The University of Agriculture, Peshawar, Pakistan.
\end{abstract}

Accepted for publication 6 January 2018.

\begin{abstract}
Fusarium head blight (FHB) is a destructive fungal disease in wheat worldwide. Efforts have been carried out to combat this disease, and the pore-forming toxin-like (PFT) gene at the quantitative trait locus (QTL) $F h b 1$ was isolated and found to confer resistance to FHB in Sumai 3. In this study, we characterized $P F T$ in 348 wheat accessions. Four haplotypes of $P F T$ were identified. The wild haplotype of $P F T$ had higher resistance than other haplotypes and explained $13.8 \%$ of phenotypic variation in FHB resistance by association analysis. PFT was highly expressed during early flowering and increased after Fusarium graminearum treatment in Sumai 3.

Analysis of the $5^{\prime}$ flanking sequence of $P F T$ predicted that the cis elements of the PFT promoter were related to hormones and biological defense responses. However, PFT existed not only in the FHB-resistant accessions but also in some susceptible accessions. These results suggested that FHB resistance in a diverse range of wheat genotypes is partially conditioned by $P F T$. The profiling of FHB resistance and the PFT locus in this large collection of wheat germplasm may prove helpful for incorporating FHB resistance into wheat breeding programs, although more work is needed to reveal the exact role of the QTL Fhb1 in conferring resistance to fungal spread.
\end{abstract}

Fusarium head blight (FHB), primarily caused by Fusarium graminearum, is one of the most destructive fungal diseases in wheat worldwide. FHB infection is favored by warm and humid conditions, especially during the flowering and early stages of kernel development in wheat (Hao et al. 2012). This disease results in severe yield losses and poor grain quality by contaminating infected grains with harmful deoxynivalenol and other trichothecene toxins (Dexter et al. 1997). These mycotoxins are a serious threat to human and animal health if consumed in food or feed products, respectively (Kazan et al. 2012; McMullen et al. 1997). Breeding for resistance to FHB in wheat is a sustainable and environmentally friendly strategy to achieve stable and cost-effective resistance (Schweiger et al. 2016).

FHB resistance is complicated by its different manifestations. Thus far, five resistance types have been identified. The first two types are most widely surveyed, including type I resistance against initial infection and type II resistance against pathogen spread within infected spikes (Schroeder and Christensen 1963). The other three types are type III, resistance to toxin accumulation; type IV, resistance to kernel infection; and type V, yield tolerance (Mesterházy 1995). Of these five types, type II resistance is regarded as the most effective and, thus, has been extensively investigated.

Resistance to FHB in wheat is controlled by multiple quantitative trait loci (QTL) affected by environmental factors with significant

${ }^{\dagger}$ Corresponding author: H. Ma; E-mail: hongxiangma@163.com

Funding: This work was partially supported by the National Key Project for the Research and Development of China (2016YFD0101802 and 2016YFE0112900), China Agricultural Research System program (CARS-03), National Natural Science Foundation of China (31561143004), Natural Science Foundation of Jiangsu Province, China (BK20170605), and European Union Horizon 2020 Mycokey project (EU678781).

Y. He and X. Zhang have contributed equally to this work.

*The $\boldsymbol{e}$-Xtra logo stands for "electronic extra" and indicates that two supplementary tables are published online.

(c) 2018 The American Phytopathological Society genotype-environment interactions (Bai and Shaner 2004; Cai et al. 2016; Ma et al. 2006; Steiner et al. 2017). To date, more than 100 QTL for FHB resistance have been reported on all 21 chromosomes in wheat (Buerstmayr et al. 2009; Steiner et al. 2017). However, most of these QTL are minor contributors to overall resistance, and only a handful of QTL have been validated and successfully employed in breeding programs worldwide (Steiner et al. 2017). Among these, the strongest and best-validated resistance QTL is $F h b 1$, which was first identified from the Chinese cultivar Sumai 3. Sumai 3 and its derivatives are considered to have a high level of type II resistance and, thus, are used as some of the most important FHB resistance donors worldwide. Fhbl, located on wheat chromosome 3BS, was first described as a strong contributor to type II resistance in two recombinant inbred populations derived from Sumai 3 and its derivative Ning 7840 using restriction fragment length polymorphism and amplified fragment length polymorphism markers separately (Bai et al. 1999; Waldron et al. 1999). This QTL was further confirmed by many other studies (Anderson et al. 2001; Buerstmayr et al. 2002; Cuthbert et al. 2006; Prat et al. 2017). Fine mapping of $F h b 1$ narrowed the original confidence interval from simple sequence repeat markers gwm493 and gwm533 to a 1.2centimorgan (cM) interval between STS3B-189 and STS3B-206 and, finally, to a 0.08-cM region flanked by the markers STS3B-355 and STS3B-334 (Anderson et al. 2001; Liu et al. 2006, 2008). Several attempts have been made to identify candidate genes in Fhbl by transcriptome-based analysis and genomic contig sequencing (Choulet et al. 2010; Schweiger et al. 2013, 2016; Xiao et al. 2013). However, functional validation of candidate genes in wheat remains challenging due to genome size and polyploidy. Recently, Bikram Gill and colleagues performed map-based cloning to identify $F h b l$ candidate genes and found that pore-forming toxin-like (PFT) confers FHB resistance by mutation analysis, gene silencing, and transgenic overexpression in wheat (Rawat et al. 2016). PFT is predicted to encode a chimeric lectin with two agglutinin domains and an EXT/ MTX2 toxin domain (Rawat et al. 2016), although this finding is still in dispute (Jia et al. 2018). Additional investigation of PFT is necessary to fully understand $F h b l$ and allow the design of robust markers for marker-assisted breeding, gene pyramiding, and genetic 
engineering to provide a long-term, economical solution to FHB outbreaks.

In the present study, we tested type II FHB resistance in 348 wheat accessions collected mainly from China using single-spikelet inoculation and further investigated the presence of PFT in these accessions. We also performed a time-course expression analysis of PFT in 12 wheat varieties with different FHB resistance levels at $8,24,48$, and $72 \mathrm{~h}$ after inoculation with $F$. graminearum. Furthermore, we examined the spatiotemporal expression pattern of $P F T$, analyzed the PFT promoter sequence and determined its expression pattern under different stimuli such as naphthylacetic acid (NAA), gibberellin (GA), abscisic acid (ABA), methyl jasmonate (MeJA), and salicylic acid (SA), as well as drought and low temperature. This study presents a comprehensive characterization of PFT in worldwide wheat germplasm and further adds to the understanding of PFT expression in response to F. graminearum and various other stimuli.

\section{MATERIALS AND METHODS}

Plant materials and growth conditions. In total, 348 wheat accessions originating from China $(n=293)$, Japan $(n=14)$, the United States $(n=7)$, Mexico $(n=6)$, Australia $(n=4)$, Canada $(n=$ $4)$, Italy $(n=3)$, and Brazil $(n=2)$ plus 15 accessions of unknown origin were used in this study (Supplementary Table S1). Plants were grown at the Jiangsu Academy of Agricultural Sciences $\left(32.0^{\circ} \mathrm{N}, 118.9^{\circ} \mathrm{E}\right)$, Nanjing, China, in the cropping seasons 2009-10, 2010-11, and 2015-16. Sumai 3 seedlings were raised in petri dishes in a growth chamber $\left(20\right.$ and $25^{\circ} \mathrm{C}$, with $16 \mathrm{~h}$ of light and $8 \mathrm{~h}$ of darkness) under light intensity of $250 \mathrm{mE} \mathrm{m}^{-2} \mathrm{~s}^{-1}$.

FHB resistance evaluation. Type II resistance was measured in 348 wheat accessions by single floret inoculation as described by Zhang et al. (2004). Approximately $10 \mu \mathrm{l}$ of $F$. graminearum fungal suspension $\left(1 \times 10^{6}\right.$ conidia/ml $)$ was injected into the central spikelets at early anthesis. The inoculated spikes were covered with a plastic bag for 3 days to meet the moisture requirement for fungal infection. The percentage of scabbed spikelets was calculated as FHB severity at 21 days after inoculation. For each accession, 10 plants were inoculated and evaluated for type II resistance.

Sumai 3 seedling treatment. Five-day-old seedlings of Sumai 3 were treated for different stimuli with $F$. graminearum suspension $\left(1 \times 10^{6}\right.$ conidia/ml $), 200 \mu \mathrm{M} \mathrm{ABA}, 100 \mu \mathrm{M}$ MeJA, $2 \mathrm{mM} \mathrm{SA}$, $50 \mu \mathrm{M}$ GA, $50 \mu \mathrm{M}$ NAA, $200 \mathrm{mM}$ sodium chloride $(\mathrm{NaCl})$, dehydration (20\% PEG6000), and low temperature $\left(4^{\circ} \mathrm{C}\right)$. Samples were harvested at $3,6,12$, and $24 \mathrm{~h}$ after treatment and immediately frozen in liquid nitrogen, followed by storage at $-80^{\circ} \mathrm{C}$ until use for further study. All experiments were repeated in biological triplicate.

RNA and DNA extraction. Total RNA was extracted from seedling, root, stem, flag leaf, and developing spikelet at different stages with the Promega SV Total RNA Isolation System (Promega Corp.), according to the manufacturer's protocol. Genomic DNA was extracted from the fresh leaves of 348 wheat accessions by using Karroten Plant Genomic DNA Extraction Kit (Karroten). The RNA and DNA quality and quantity were determined by agarose gel electrophoresis and NanoDrop 2000 (Thermo Scientific).

Quantitative real-time polymerase chain reaction analysis. First-strand cDNA was synthesized using M-MLV Reverse Transcriptase (Fermentas). Quantitative real-time polymerase chain reaction (PCR) analyses were performed on a CFX96 (BioRad) using a SYBR Green detection protocol according to the manufacturer's instructions. The tubulin gene was used as an endogenous control gene (Supplementary Table S2). PFT-RTF and PFT-RTR primers were designed for PFT gene analysis. The PCR conditions were $95^{\circ} \mathrm{C}$ for $30 \mathrm{~s}, 95^{\circ} \mathrm{C} 5 \mathrm{~s}, 60^{\circ} \mathrm{C}$ for $20 \mathrm{~s}$, and $72^{\circ} \mathrm{C}$ for $10 \mathrm{~s}$ for 45 cycles; followed by $95^{\circ} \mathrm{C}$ for $10 \mathrm{~s}, 65^{\circ} \mathrm{C}$ for $10 \mathrm{~s}$, and $95^{\circ} \mathrm{C}$ for $5 \mathrm{~s}$. Data from the individual runs were collated using the $2^{-\Delta \Delta \mathrm{CT}}$ method (Livak and Schmittgen 2001). All reactions were performed in three separate experiments.

\section{RESULTS}

Gene amplification of $P F T$ in 348 wheat accessions. The $P F T$ gene at the $F h b 1$ locus was previously reported to confer FHB resistance in Sumai 3 and was detected in 21 other resistant lines (Rawat et al. 2016). To gain a broad characterization of PFT in wheat, we collected 348 wheat accessions in the present study, mainly from China and several other countries such as Japan, the United States, and Mexico. We designed three pairs of primers covering the entire $P F T$ open reading frame and part of the promoter and intron regions (Fig. 1A) based on the reported gene sequence (Rawat et al. 2016). Among all the tested wheat genotypes, the PFT was amplified in 164 accessions but not in the remaining 184 accessions (Fig. 1B).

Identification of single-nucleotide polymorphisms and haplotypes at the $P F T$ locus. To assay single-nucleotide polymorphisms (SNP) in PFT, we sequenced all 164 PFT-positive wheat accessions by using the abovementioned three primers. Among these accessions, we found two haplotypes different from that of Sumai 3 (referred to as the wild type) (Fig. 2). One haplotype (S1) carried three SNP, two of which were the same as those described by the previous study, including a silent change (T/C) and an SNP (G/A) that caused aberrant mRNA splicing of mRNA (Rawat et al. 2016). In addition to the abovementioned two SNP, we also identified a new silent SNP (A/G) (Fig. 2). In addition, a novel haplotype (S2) was found and contained a new SNP $(-/ G)$ that caused a frameshift and premature translational termination (Fig. 2). HapS1 was found in 19 wheat accessions, including Nanda 2419, Zhengmai 366, Jimai 20, Wenmai 8, Huaimai 18, Yumai 49, Shanmai 150, Zhenmai 6, Laizhou 187, and CA 25. Hap-S2 occurred in only four accessions: Xinong 6426, Ziganzi, Baihuomai, and Beinuo 1.

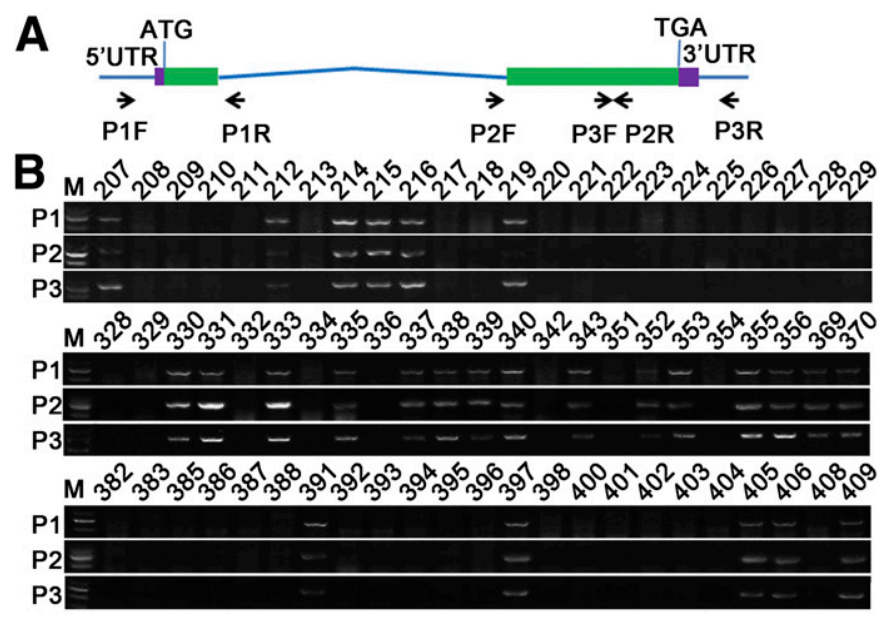

Fig. 1. Characterization of the pore-forming toxin-like (PFT) gene in wheat accessions. A, Location of the three pairs of primers used for identifying the PFT gene. UTR = untranslated region. B, Partial results of the polymerase chain reaction amplification of $P F T$ in 348 wheat accessions.

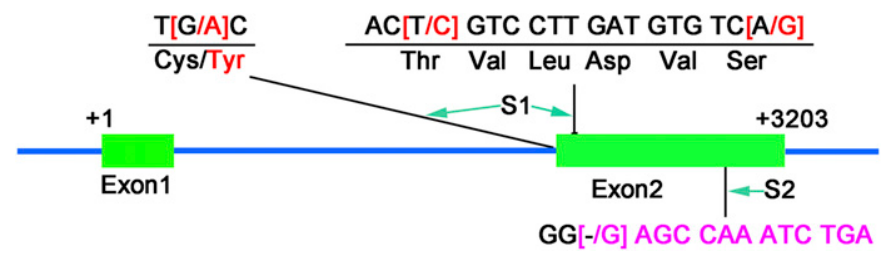

Fig. 2. Location of single nucleotide polymorphisms (SNP) within the poreforming toxin-like (PFT) gene. Coding sequence is depicted as the thick horizontal line. Two haplotypes distinct from that of Sumai 3 (S1 and S2) are depicted as capital letters above and below the horizontal line, respectively. 
FHB resistance in 348 wheat accessions. FHB resistance in 348 wheat accessions was evaluated by using single-spikelet inoculation as described previously (Zhang et al. 2004). FHB severity showed continuous variation among the 348 accessions, with a major peak in the number of accessions with proportion of symptomatic spikelets (PSS) ranging from 30 to $40 \%$ (Fig. 3A). Wheat responses to FHB infection can be divided into four levels according to PSS: resistant (PSS $<25 \%$, such as Sumai 3 and Wangshuibai), moderately resistant $(25 \%<\mathrm{PSS}<50 \%$, such as Ningmai 3 and Aso Zairai II), moderately susceptible $(50 \%<\mathrm{PSS}<75 \%$, such as Xiaoyan 54 and Clark), and susceptible (PSS $>75 \%$, such as Nanda 2419 and Annong 8455).

PFT haplotypes associated with FHB resistance. Four haplotypes were found in the 348 accessions by the above sequence analysis: Hap-W, Hap-S1, Hap-S2, and Hap-N. The average PSS was $36.9 \%$ in the Hap-W wheat accessions, which carried the wildtype PFT sequence, whereas the average PSS were $55.7 \%$ and $84.9 \%$ for accessions that carried the S1 and S2 SNP variation, respectively, in this study (Fig. 3B). In the Hap-N accessions, which did not carry PFT, the average PSS was $52.5 \%$ (Fig. 3B). Association analysis showed that Hap-W, Hap-S2, and Hap-N were significantly associated with FHB severity. Hap-W could explain $13.8 \%$ of the phenotypic variation (Table 1 ).

Expression analysis of $P F T$ in 12 wheat varieties after inoculation with $\boldsymbol{F}$. graminearum. $P F T$ was reported to exist only in Fhbl-resistant lines (Rawat et al. 2016). However, from the

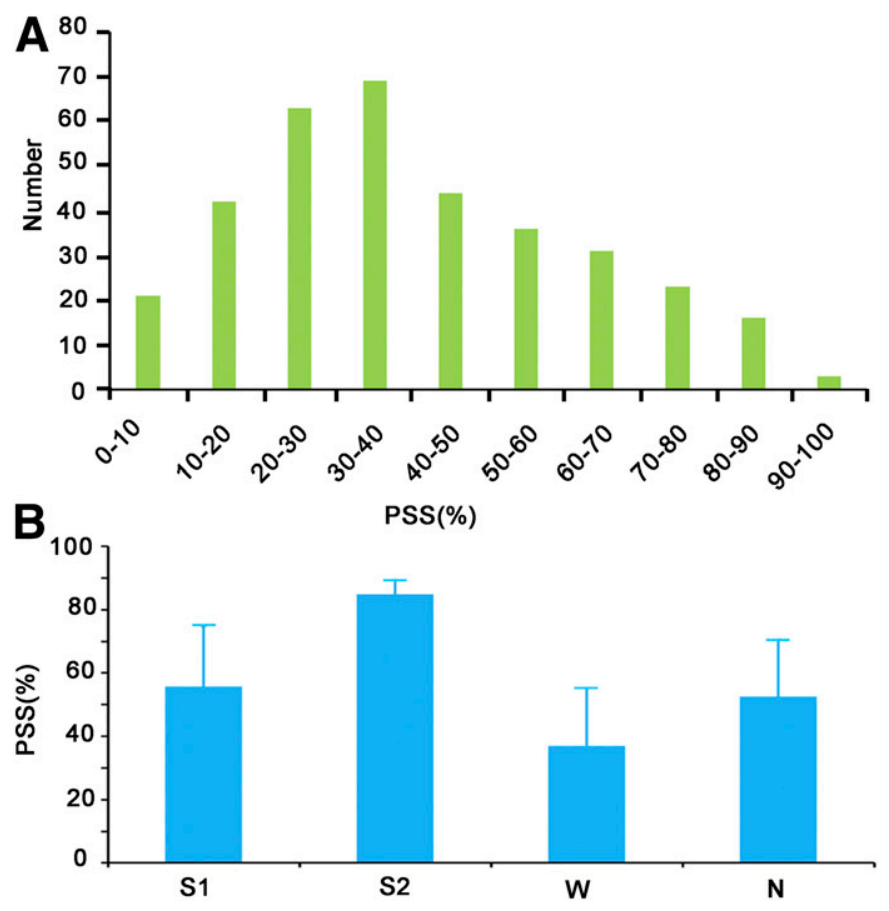

Fig. 3. Type II Fusarium head blight (FHB) resistance and pore-forming toxinlike $(P F T)$ haplotypes associated with their FHB phenotypes. A, Distribution curve of the type II FHB resistance in 348 wheat accessions. B, FHB phenotypes are reflected by the average proportion of symptomatic spikelets (PSS) in S1, S2, wild-type (W), and null-PFT (N) accessions.

TABLE 1. Pore-forming toxin-like haplotypes associated with the resistance to Fusarium head blight (FHB)

\begin{tabular}{lcc}
\hline Haplotype & $P$ value & PVE $(\%)^{\mathrm{a}}$ \\
\hline Hap-S1 & 0.013560901 & 2.1 \\
Hap-S2 & $6.04 \mathrm{E}-05$ & 5.5 \\
Hap-W & $8.18 \mathrm{E}-11$ & 13.9 \\
Hap-N & $1.76 \mathrm{E}-05$ & 6.3 \\
\hline
\end{tabular}

a $\mathrm{PVE}=$ phenotypic variation explained. above results, we found that it was also present in many susceptible wheat varieties such as Guandong $107(75.29 \pm 21.93 \%)$ and Chuannong $42(79.47 \pm 15.75 \%)$. To test whether there was any difference in PFT expression pattern between the resistant and susceptible lines after F. graminearum inoculation, we randomly chose 12 wheat varieties with different FHB resistance levels, including all three PFT-positive haplotypes (Fig. 4). Among the wild-type varieties, PFT was upregulated in all eight selected lines, although the upregulation occurred at different times (Fig. 4A to $\mathrm{H}$ ). Different expression patterns were found in the Hap-S1 and Hap-S2 wheat varieties. PFT was upregulated in Zhenmai 6 (S1) and Xinong 6426 (S2) but not in Yuandong 107 (S1) or Huaimai 18 (S1) (Fig. 4I to L).

Spatiotemporal expression pattern of PFT in Sumai 3. $P F T$ has been found to play a critical role in conferring FHB resistance in Sumai 3 (Rawat et al. 2016). To investigate its spatiotemporal expression pattern in Sumai 3, quantitative reverse-transcription (qRT)PCR analyses in 5-day-old seedling, root, stem, flag leaf, and developing spikelets at different stages were performed as shown in Figure 5. The stages of the spikelets were classified based on the previous description ( $\mathrm{Li}$ et al. 2017). The results showed that PFT transcript levels were low in root, stem, and flag leaf; high in the seedling and early stages of spikelet development; and reached a maximum at the microspore mother cell stage; afterward gradually declining to a low level at the anthesis stage (Fig. 5).

Analysis of the 5'-flanking sequence of PFT. For a more comprehensive understanding of cis-acting regulatory elements, the 2,999-bp upstream sequence before the translation initiation codon (KX907434) was analyzed using the PlantCARE database (Lescot et al. 2002). The bioinformatics analysis of the PFT promoter detected many core cis-acting regulatory elements (Table 2). For example, one fungal elicitor response (Box_W1: TTGACC), four kinds of stress response (HSE: AAAAAATTTC, LTR: CCGAAA, TC-rich repeats: ATTTTCTCCA, and GC-motif: CCCCCG) and three MYB binding-site (MBS: TAACTG, MBS: CGGTCA, and CCAAT-box: CAACGG) cis elements were found. Furthermore, cis-acting elements related to hormones were also predicted. For example, four ABA response (ABRE: CACGTG), six MeJA response (CGTCA-motif: CGTCA and TGACG-motif: TGACG), one GA response (P-box: CCTTTTG), one SA response (TCA element: $G$ AGAAGAATA), and one auxin response (TGA element: AACGAC) motif were detected. Overall, the prediction results showed that the regulatory cis elements of the PFT promoter were related to hormones and stress-induced responses, indicating that the promoter may play an important role in response to external environmental stresses and biological defense processes.

$P F T$ expression analysis in response to different stimuli. qRT-PCR was carried out in 5-day-old Sumai 3 seedlings treated with $F$ graminearum and hormones, including ABA, SA, GA3, NAA, and MeJA. The results showed that PFT expression was increased due to $F$. graminearum inoculation (Fig. 6A) and increased significantly when treated with MeJA after $6 \mathrm{~h}$ posttreatment (hpt) compared with the control, and reached its strongest response at $6 \mathrm{hpt}$ (Fig. 6C). A similar pattern was revealed under ABA treatment, where the PFT transcript level reached its highest level at $12 \mathrm{hpt}$ (Fig. 6E). PFT expression gradually increased under SA and NAA treatment (Fig. 6D and $F$ )and showed a slight increase under GA3 treatment before $12 \mathrm{hpt}$ but decreased at $24 \mathrm{hpt}$ (Fig. 6B). To determine the function of PFT in responses to abiotic stress, we investigated the time-course expression patterns of PFT in Sumai 3 seedlings under $\mathrm{NaCl}$, cold $\left(4^{\circ} \mathrm{C}\right)$, and drought (PEG6000) treatments. PFT mRNA was enhanced by $\mathrm{NaCl}$ treatment but was only slightly affected by drought (Fig. 6G and $\mathrm{H}$ ). When seedlings were treated with low temperature, the PFT transcript level gradually increased over time (Fig. 6I).

\section{DISCUSSION}

Resistance to FHB in wheat is a quantitatively inherited trait. Several types of FHB resistance have been described; type II 
resistance is regarded as the most effective and, thus, has been extensively investigated (Steiner et al. 2017). Molecular mapping results showed that the major QTL $F h b 1$ on the short arm of chromosome 3B was the strongest and best validated QTL for type II resistance in wheat, especially in the resistant varieties originating from Asia (Bai and Shaner 2004). Extensive efforts have been made to identify FHB-responsive candidate genes in the Fhbl QTL in wheat (Schweiger et al. 2013, 2016; Xiao et al. 2013; Zhang et al. 2013; Zhuang et al. 2013). PFT at the Fhbl locus has been identified to confer type II FHB resistance by mutation analysis, gene silencing, and transgenic overexpression in wheat (Rawat et al. 2016). PFT is predicted to encode a chimeric lectin with two agglutinin domains and an ETX/MTX2 toxin domain. However, the role of PFT in FHB resistance is still disputed (Jia et al. 2018).

In the present study, PFT haplotypes and FHB resistance phenotypes were characterized in a large collection of 348 diverse wheat accessions that encompassed a wide range of FHB resistance responses. The germplasm mostly included landraces and breeding varieties from China, as well as accessions from some other countries, such as Japan, the United States, and Mexico. The PFT sequence was amplified in only 164 accessions whereas, in the remaining 184 accessions, $P F T$ was not detected. To make sure the absence was not due to the negative PCR results, we designed three primer pairs to amplify products covering the whole sequence of PFT (Rawat et al. 2016). The first PCR product amplified $188 \mathrm{bp}$ of promoter, the $5^{\prime}$ untranslated region (UTR), the exon 1, and $193 \mathrm{bp}$ of the intron. The second product got $80 \mathrm{bp}$ of the intron and $128 \mathrm{bp}$ of exon 2. The third product contained the rest of exon 2 with $80 \mathrm{bp}$ of overlapped fragment and the $3^{\prime} \mathrm{UTR}$. All of these products were consistently not detected in the remaining 184 accessions, which largely confirmed the absence of the PFT.

In the previous study, one resistant and one susceptible haplotype were found for PFT (Rawat et al. 2016). Of the 40 wheat landraces and cultivars they sequenced, all 22 resistant lines carried $P F T$, while 15 susceptible genotypes were null for $P F T$ and the remaining 3 genotypes carried the same two SNP, including a silent change and an SNP causing aberrant mRNA splicing (Rawat et al. 2016). In this study, two distinct haplotypes (S1 and S2) used in comparing with the wild type (Sumai 3) were identified in 164 PFT-positive accessions. A new silent SNP (A/G) along with the two previously reported SNP was identified in S1. In addition, a novel haplotype, S2, was found with a new SNP $(-/ \mathrm{G})$, leading to frameshift and premature translational termination.

FHB severity showed continuous variation among the 348 accessions in this study. Wheat accessions carrying the wild-type PFT sequence showed the highest resistance to FHB, with an average PSS of $36.9 \%$, whereas the average PSS were 55.7 and $84.9 \%$ in $\mathrm{S} 1$ and S2 haplotypes, respectively. Association analysis also showed that the wild haplotype had the highest explanation of phenotypic variation among the haplotypes. However, unlike previous researchers, we found that the wild-type PFT sequence

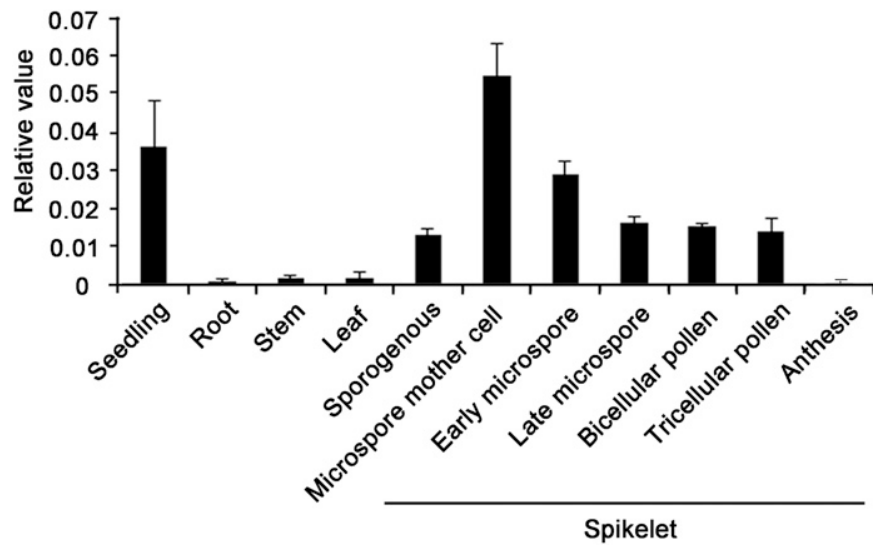

Fig. 5. Expression pattern of the pore-forming toxin-like gene in 5-day-old seedling, root, stem, flag leaf, and spikelets at different stages in Sumai 3. The tubulin gene was used as an internal control. Values shown represent the means of three biological experiments and error bars indicate the standard errors of the mean.
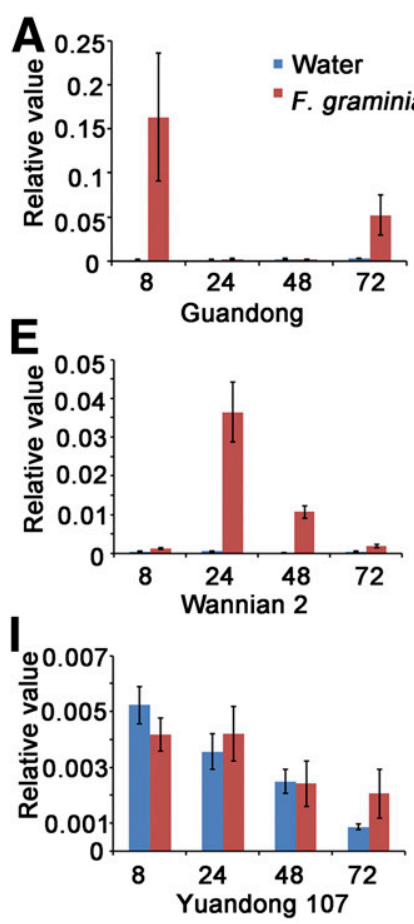

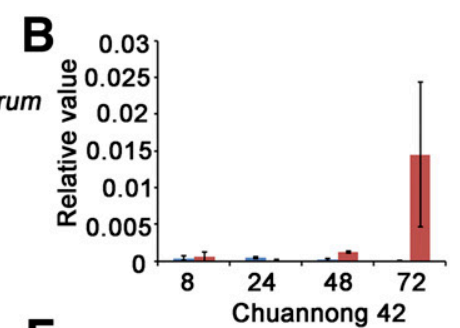

$\mathbf{F}$
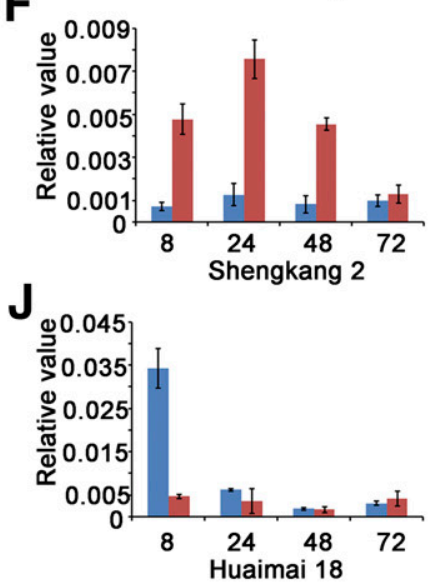

\section{C}

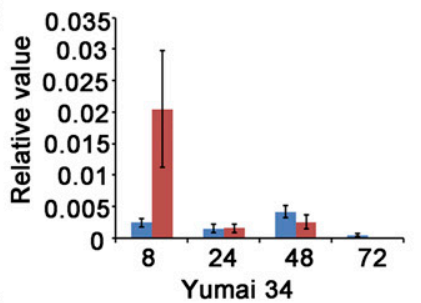

G

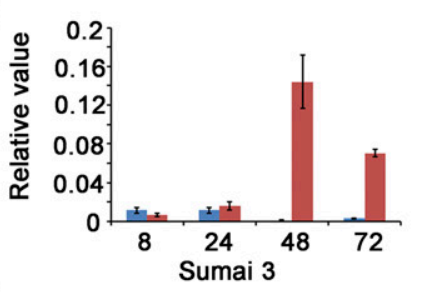

$\mathbf{K}$

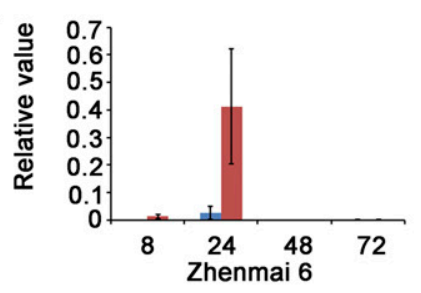

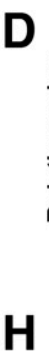
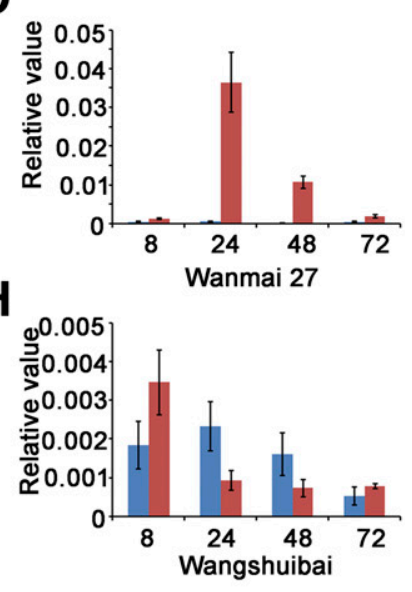

$\mathbf{L}$

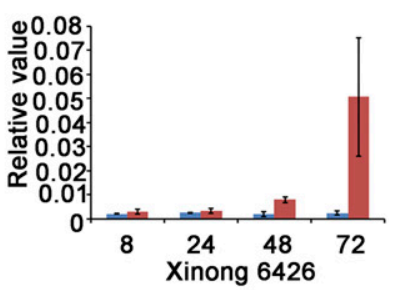

Fig. 4. Expression analysis of pore-forming toxin-like $(P F T)$ in 12 wheat varieties after inoculation with Fusarium graminearum. A to $\mathbf{H}$, Wheat accessions carrying the wild-type PFT sequence with different Fusarium head blight resistance levels. I to K, Haplotype S1 wheat accessions. L, Haplotype S2 wheat accession. 
existed not only in resistant accessions but also in susceptible accessions such as Nonglin 67, Chuannong 42, and Xiaoyan 54. Furthermore, five moderately resistant accessions such as CA25, Ningyan 1, and Zhenmai 6 had mutant instead of wild-type PFT sequences. For the 184 PFT-negative accessions, only 102 accessions were susceptible to FHB, whereas the remaining 82 accessions demonstrated moderate or high FHB resistance. Furthermore, PFT could be upregulated in both resistant and susceptible

TABLE 2. Putative cis-acting regulatory element analysis of the pore-forming toxin-like (PFT) promoter sequence by PlantCARE

\begin{tabular}{|c|c|c|c|c|}
\hline Motif & Location & Strand $^{\mathrm{a}}$ & Sequence & Putative function $^{\mathrm{b}}$ \\
\hline ABRE & $-368,-1431,-1907,-2915$ & - & CACGTG & $\begin{array}{l}\text { cis-Acting element involved in the abscisic } \\
\text { acid response }\end{array}$ \\
\hline CGTCA-motif & $-1570,-2203,-2580$ & + & CGTCA & $\begin{array}{l}\text { cis-Acting regulatory element involved in } \\
\text { the MeJA-response }\end{array}$ \\
\hline TGACG-motif & $-1570,-2203,-2580$ & + & TGACG & $\begin{array}{l}\text { cis-Acting regulatory element involved in } \\
\text { the MeJA-response }\end{array}$ \\
\hline P-box & -1331 & + & CCTTTTG & Gibberellin-responsive element \\
\hline TCA element & -450 & - & GAGAAGAATA & $\begin{array}{l}\text { cis-Acting element involved in salicylic acid } \\
\text { response }\end{array}$ \\
\hline TGA element & -2216 & + & AACGAC & Auxin-responsive element \\
\hline Box-W1 & -1202 & + & TTGACC & Fungal elicitor responsive element \\
\hline HSE & -2293 & - & AAAAAATTTC & $\begin{array}{l}\text { cis-Acting element involved in heat stress } \\
\text { response }\end{array}$ \\
\hline LTR & $-932,-2996,-2977$ & - & CCGAAA & $\begin{array}{l}\text { cis-Acting element involved in low- } \\
\text { temperature response }\end{array}$ \\
\hline TC-rich repeats & -754 & + & ATTTTCTCCA & $\begin{array}{l}\text { cis-Acting element involved in defense and } \\
\text { stress response }\end{array}$ \\
\hline GC-motif & $-2028,-2169,-2904,-2934$ & - & CCCCCG & $\begin{array}{l}\text { Enhancer-like element involved in anoxic } \\
\text { specific inducibility }\end{array}$ \\
\hline MBS & $-1193,-1913$ & - & TAACTG & $\begin{array}{l}\text { MYB binding site involved in drought- } \\
\text { inducibility }\end{array}$ \\
\hline MBS & -1201 & - & CGGTCA & MYB binding site \\
\hline CCAAT-box & $-1017,-1123$ & - & CAACGG & MYBHv1 binding site \\
\hline
\end{tabular}

a Sense (+) and antisense (-) strands.

b MeJA = methyl jasmonate.
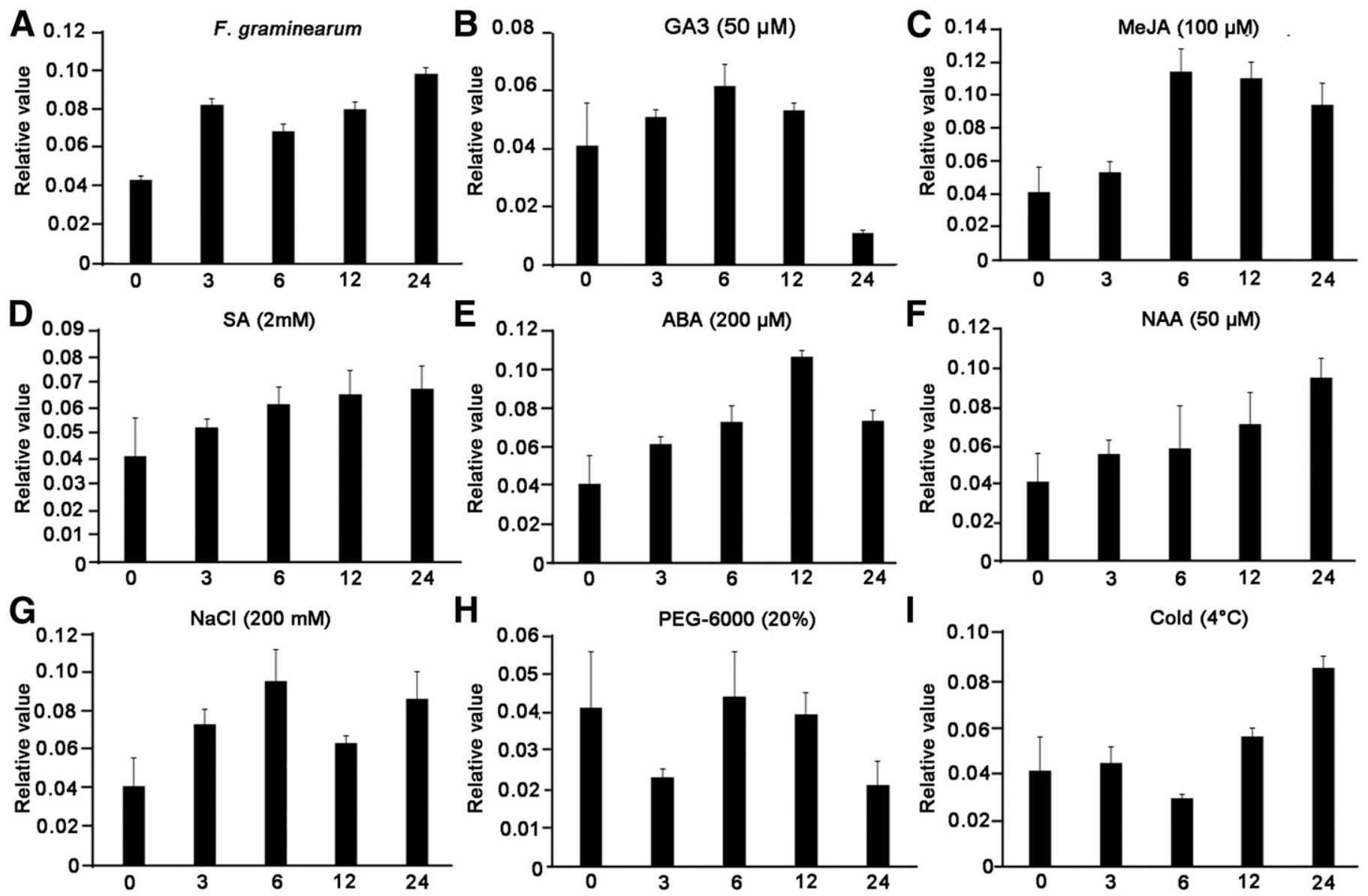

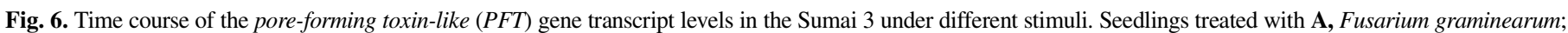

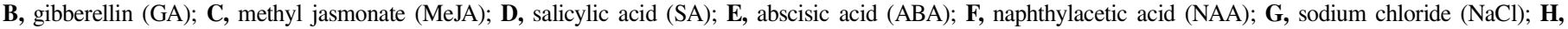

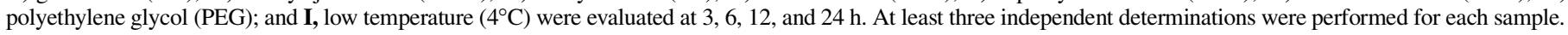


accessions. We speculate that wheat resistance to FHB is a multigenic complex trait, and PFT represents one of the genes that assist FHB resistance. Additional efforts are still needed to reveal the mechanisms of Fhbl in FHB resistance.

Gene expression is transcriptionally regulated by a change in the level or activity of sequence-specific DNA-binding transcription factors bound to specific cis-acting elements of promoter regions (Liao et al. 2015). These regulatory factors are involved in the activation, suppression, and modulation of various signaling pathways responsive to biotic and abiotic stress in plants. Therefore, many plant promoters have been identified and isolated, and genetic engineering in plants has been greatly enhanced using individual promoters. The bioinformatics analysis showed that multiple cis-acting elements, including elements associated with fungal elicitor response, abiotic stresses, and hormone regulation, occur in the PFT promoter, indicating that the $P F T$ gene might respond to various environmental stimuli.

PFT transcription increased in the spikelet of Sumai 3 after inoculation with $F$. graminearum macroconidia, as described previously (Rawat et al. 2016). Furthermore, PFT transcription also increased following $F$. graminearum inoculation during the seedling stage. Phytohormones serve as important signaling molecules and play crucial roles in controlling the expression of downstream defense genes and physiological reactions to various stresses. SA and JA signaling in the wheat FHB disease response has been well characterized (Gottwald et al. 2012; Li and Yang 2008; Qi et al. 2012, 2016). Increases in the levels of MeJA and SA in infected spikes were shown to correlate with FHB resistance (Li and Yang 2008; Makandar et al. 2012). Using combined proteomic and transcriptomic approaches, FHB resistance was found to be associated with the coordinated and ordered expression of diverse defense pathways involving signaling molecules such as SA and JA (Ding et al. 2011; Xiao et al. 2013). In our results, MeJA induced a significant increase in $P F T$ transcription whereas SA treatment resulted in a smaller increase. The results indicate that PFT may play a role in JA-mediated FHB resistance in wheat, consistent with the fact that JA plays a more important role than SA in responses to necrotrophic and hemibiotrophic pathogens.

In addition to the classical defense response phytohormones, ABA, GA, and auxin have also been shown to modulate pathogenesis in wheat, with GA and auxin promoting resistance and ABA promoting susceptibility (Buhrow et al. 2016; Gordon et al. 2016; Petti et al. 2012). PFT expression gradually increased under NAA treatment, and it showed a slight increase under GA3 treatment before $12 \mathrm{hpt}$ but decreased at $24 \mathrm{hpt}$. PFT transcription significantly increased with $\mathrm{ABA}$ treatment, suggesting a complex interaction among phytohormones in the FHB response. PFT might also play a role in stress-related signaling pathways because it was induced by $\mathrm{NaCl}$ and cold-temperature treatments.

In conclusion, this study presents a thorough, sequence-based description of $P F T$ in a larger number of wheat accessions. It was shown that $P F T$ is one of the contributors to type II resistance to FHB. A novel SNP $(-/ \mathrm{G})$ causing a frameshift and premature translational termination was identified in FHB-susceptible accessions. PFT was highly expressed during early flower development and increased after $F$. graminearum treatment. Multiple cis-acting elements, including elements associated with abiotic stresses and hormone regulation, were found in the $P F T$ promoter by bioinformatics analysis. Expression pattern analysis suggested that $P F T$ might play a role in tolerance to salt and cold stresses and in defense responses by mediating ABA, SA, MeJA, and NAA signaling. The FHB resistance and PFT locus profiling of this large collection of wheat germplasm may prove helpful in incorporating FHB resistance in future wheat breeding programs, although more work must to be done to confirm the exact role of PFT in conferring resistance to fungal spread.

\section{LITERATURE CITED}

Anderson, J. A., Stack, R. W., Liu, S., Waldron, B. L., Fjeld, A. D., Coyne, C., Morenosevilla, B., Fetch, J. M., Song, Q. J., and Cregan, P. B. 2001. DNA markers for Fusarium head blight resistance QTLs in two wheat populations. Theor. Appl. Genet. 102:1164-1168.

Bai, G., Kolb, F. L., Shaner, G., and Domier, L. L. 1999. Amplified fragment length polymorphism markers linked to a major quantitative trait locus controlling scab resistance in wheat. Phytopathology 89:343-348.

Bai, G., and Shaner, G. 2004. Management and resistance in wheat and barley to Fusarium head blight. Annu. Rev. Phytopathol. 42:135-161.

Buerstmayr, H., Ban, T., and Anderson, J. A. 2009. QTL mapping and markerassisted selection for Fusarium head blight resistance in wheat: A review. Plant Breed. 128:1-26.

Buerstmayr, H., Lemmens, M., Hartl, L., Doldi, L., Steiner, B., Stierschneider, M., and Ruckenbauer, P. 2002. Molecular mapping of QTLs for Fusarium head blight resistance in spring wheat. I. Resistance to fungal spread (type II resistance). Theor. Appl. Genet. 104:84-91.

Buhrow, L. M., Cram, D., Tulpan, D., Foroud, N. A., and Loewen, M. C. 2016. Exogenous abscisic acid and gibberellic acid elicit opposing effects on Fusarium graminearum infection in wheat. Phytopathology 106:986-996.

Cai, J., Wang, S., Li, T., Zhang, G., and Bai, G. 2016. Multiple minor QTLs are responsible for Fusarium head blight resistance in Chinese wheat landrace Haiyanzhong. PLoS One 11:e0163292.

Choulet, F., Wicker, T., Rustenholz, C., Paux, E., Salse, J., Leroy, P., Schlub, S., Le Paslier, M. C., Magdelenat, G., Gonthier, C., Couloux, A., Budak, H., Breen, J., Pumphrey, M., Liu, S., Kong, X., Jia, J., Gut, M., Brunel, D., Anderson, J. A., Gill, B. S., Appels, R., Keller, B., and Feuillet, C. 2010. Megabase level sequencing reveals contrasted organization and evolution patterns of the wheat gene and transposable element spaces. Plant Cell 22: 1686-1701.

Cuthbert, P. A., Somers, D. J., Thomas, J., Cloutier, S., and Brule-Babel, A. 2006. Fine mapping $F h b 1$, a major gene controlling Fusarium head blight resistance in bread wheat (Triticum aestivum L.). Theor. Appl. Genet. 112: 1465-1472.

Dexter, J. E., Marchylo, B. A., Clear, R. M., and Clarke, J. M. 1997. Effect of Fusarium head blight on semolina milling and pasta-making quality of durum wheat. Cereal Chem. 74:519-525.

Ding, L., Xu, H., Yi, H., Yang, L., Kong, Z., Zhang, L., Xue, S., Jia, H., and Ma, Z. 2011. Resistance to hemi-biotrophic $F$. graminearum infection is associated with coordinated and ordered expression of diverse defense signaling pathways. PLoS One 6:e19008.

Gordon, C. S., Nandhakishore, R., Risseeuw, E. P., Marci, S., Ball, F. J., Barber, C. J., Buhrow, L. M., Clark, S. M., Page, J. E., and Todd, C. D. 2016. Characterization of Triticum aestivum abscisic acid receptors and a possible role for these in mediating Fusarium head blight susceptibility in wheat. PLoS One 11:e0164996.

Gottwald, S., Samans, B., Luck, S., and Friedt, W. 2012. Jasmonate and ethylene dependent defence gene expression and suppression of fungal virulence factors: Two essential mechanisms of Fusarium head blight resistance in wheat? BMC Genomics 13:369.

Hao, C., Wang, Y., Hou, J., Feuillet, C., Balfourier, F., and Zhang, X. 2012. Association mapping and haplotype analysis of a $3.1-\mathrm{Mb}$ genomic region involved in Fusarium head blight resistance on wheat chromosome 3BS. PLoS One 7:e46444.

Jia, H., Zhou, J., Xue, S., Li, G., Yan, H., Ran, C., Zhang, Y., Shi, J., Jia, L., Wang, X., Luo, J., and Ma, Z. 2018. A journey to understand wheat Fusarium head blight resistance in the Chinese wheat landrace Wangshuibai. Crop J. 6:48-59.

Kazan, K., Gardiner, D. M., and Manners, J. M. 2012. On the trail of a cereal killer: Recent advances in Fusarium graminearum pathogenomics and host resistance. Mol. Plant Pathol. 13:399-413.

Lescot, M., Déhais, P., Thijs, G., Marchal, K., Moreau, Y., Peer, Y. V. D., Rouzé, P., and Rombauts, S. 2002. PlantCARE, a database of plant cisacting regulatory elements and a portal to tools for in silico analysis of promoter sequences. Nucleic Acids Res. 30:325-327.

Li, D. X., Hu, H. Y., Li, G., Ru, Z. G., and Tian, H. Q. 2017. Calcium controls the formation of vacuoles from mitochondria to regulate microspore development in wheat. Plant Reprod. 30:131-139.

Li, G., and Yang, Y. 2008. Jasmonate and ethylene signaling pathway may mediate Fusarium head blight resistance in wheat. Crop Sci. 48:1888-1896.

Liao, Y. L., Shen, Y. B., Chang, J., Zhang, W. W., Cheng, S. Y., and Xu, F. 2015. Isolation, expression, and promoter analysis of GbWRKY2: A novel transcription factor gene from Ginkgo biloba. Int. J. Genomics 2015: Article 607185.

Liu, S., Pumphrey, M. O., Gill, B. S., Trick, H. N., Zhang, J. X., Dolezel, J., Chalhoub, B., and Anderson, J. A. 2008. Toward positional cloning of Fhb1, a major QTL for Fusarium head blight resistance in wheat. Cereal Res. Commun. 36:195-201.

Liu, S., Zhang, X., Pumphrey, M. O., Stack, R. W., Gill, B. S., and Anderson, J. A. 2006. Complex microcolinearity among wheat, rice, and barley revealed by fine mapping of the genomic region harboring a major QTL for resistance to Fusarium head blight in wheat. Funct. Integr. Genomics 6:83-89. 
Livak, K. J., and Schmittgen, T. D. 2001. Analysis of relative gene expression data using real-time quantitative PCR and the $2^{-\Delta \Delta C T}$. Methods 25:402-408.

Ma, H. X., Bai, G. H., Zhang, X., and Lu, W. Z. 2006. Main effects, epistasis, and environmental interactions of quantitative trait loci for Fusarium head blight resistance in a recombinant inbred population. Phytopathology 96: 534-541.

Makandar, R., Nalam, V. J., Lee, H., Trick, H. N., Dong, Y., and Shah, J. 2012. Salicylic acid regulates basal resistance to Fusarium head blight in wheat. Mol. Plant-Microbe Interact. 25:431-439.

McMullen, M., Jones, R., and Gallenberg, D. 1997. Scab of wheat and barley: A re-emerging disease of devasting impact. Plant Dis. 81:1340-1348.

Mesterházy, A. 1995. Types and components of resistance to Fusarium head blight of wheat. Plant Breed. 114:377-386.

Petti, C., Reiber, K., Ali, S. S., Berney, M., and Doohan, F. M. 2012. Auxin as a player in the biocontrol of Fusarium head blight disease of barley and its potential as a disease control agent. BMC Plant Biol. 12:224.

Prat, N., Guilbert, C., Prah, U., Wachter, E., Steiner, B., Langin, T., Robert, O., and Buerstmayr, H. 2017. QTL mapping of Fusarium head blight resistance in three related durum wheat populations. Theor. Appl. Genet. 130:13-27.

Qi, P. F., Balcerzak, M., Rocheleau, H., Leung, W., Wei, Y. M., Zheng, Y. L., and Ouellet, T. 2016. Jasmonic acid and abscisic acid play important roles in host-pathogen interaction between Fusarium graminearum and wheat during the early stages of Fusarium head blight. Physiol. Mol. Plant Pathol. 93:39-48.

Qi, P. F., Johnston, A., Balcerzak, M., Rocheleau, H., Harris, L. J., Long, X. Y., Wei, Y. M., Zheng, Y. L., and Ouellet, T. 2012. Effect of salicylic acid on Fusarium graminearum, the major causal agent of Fusarium head blight in wheat. Fungal Biol. 116:413-426.

Rawat, N., Pumphrey, M. O., Liu, S., Zhang, X., Tiwari, V. K., Ando, K., Trick, H. N., Bockus, W. W., Akhunov, E., Anderson, J. A., and Gill, B. S. 2016. Wheat $F h b 1$ encodes a chimeric lectin with agglutinin domains and a pore-forming toxin-like domain conferring resistance to Fusarium head blight. Nat. Genet. 48:1576-1580.
Ren, L., Shen, X., Zhou, M., Zhang, X., Ma, H., Lu, W., and Nichoson, P. 2003. SSR markers for Fusarium head blight resistance QTLs in three wheat populations. J. Integr. Agric. 2:987-993.

Schroeder, H. W., and Christensen, J. J. 1963. Factors affecting resistance of wheat to scab caused by Gibberella zeae. Phytopathology 53:831-838.

Schweiger, W., Steiner, B., Ametz, C., Siegwart, G., Wiesenberger, G., Berthiller, F., Lemmens, M., Jia, H., Adam, G., and Muehlbauer, G. J. 2013. Transcriptomic characterization of two major Fusarium resistance quantitative trait loci (QTLs), Fhb1 and Qfhs.ifa-5A, identifies novel candidate genes. Mol. Plant Pathol. 14:772-785.

Schweiger, W., Steiner, B., Vautrin, S., Nussbaumer, T., Siegwart, G., Zamini, M., Jungreithmeier, F., Gratl, V., Lemmens, M., Mayer, K. F., Berges, H., Adam, G., and Buerstmayr, H. 2016. Suppressed recombination and unique candidate genes in the divergent haplotype encoding Fhbl, a major Fusarium head blight resistance locus in wheat. Theor. Appl. Genet. 19:1607-1623.

Steiner, B., Buerstmayr, M., Michel, S., Schweiger, W., Lemmens, M., and Buerstmayr, H. 2017. Breeding strategies and advances in line selection for Fusarium head blight resistance in wheat. Trop. Plant Pathol. 42:165-174.

Waldron, B. L., Moreno-Sevilla, B., Anderson, J. A., Stack, R. W., and Frohberg, R. C. 1999. RFLP mapping of QTL for Fusarium head blight resistance in wheat. Crop Sci. 39:805-811.

Xiao, J., Jin, X., Jia, X., Wang, H., Cao, A., Zhao, W., Pei, H., Xue, Z., He, L., and Chen, Q. 2013. Transcriptome-based discovery of pathways and genes related to resistance against Fusarium head blight in wheat landrace Wangshuibai. BMC Genomics 14:197.

Zhang, X., Fu, J., Hiromasa, Y., Pan, H., and Bai, G. 2013. Differentially expressed proteins associated with Fusarium head blight resistance in wheat. PLoS One 8:e82079.

Zhang, X., Zhou, M., Ren, L., Bai, G., Ma, H., Scholten, O. E., Guo, P., and Lu, W. 2004. Molecular characterization of Fusarium head blight resistance from wheat variety Wangshuibai. Euphytica 139:59-64.

Zhuang, Y., Gala, A., and Yen, Y. 2013. Identification of functional genic components of major Fusarium head blight resistance quantitative trait loci in wheat cultivar Sumai 3. Mol. Plant-Microbe Interact. 26:442-450. 